\title{
Characterizing Everyday Objects using Human Touch: Thermal Dissipation as a Sensing Modality
}

\section{Emenike, Hilary}

IEEE

2021

Emenike , H , Dar , F , Liyanage , M , Sharma , R, Zuniga , A, Hoque , M A, Radeta , M , Nurmi , P \& Flores , H 2021 , Characterizing Everyday Objects using Human Touch:

Thermal Dissipation as a Sensing Modality . in 2021 IEEE International Conference on Pervasive Computing and Communications (PerCom). International Conference on Pervasive Computing and Communications, IEEE, IEEE International Conference on Pervasive Computing and Communications, Kessel , Germany , 22/03/2021 . https://doi.org/10.1109/PERCOM5058

http://hdl.handle.net/10138/333648

https://doi.org/10.1109/PERCOM50583.2021.9439120

acceptedVersion

Downloaded from Helda, University of Helsinki institutional repository.

This is an electronic reprint of the original article.

This reprint may differ from the original in pagination and typographic detail.

Please cite the original version. 


\title{
Characterizing Everyday Objects using Human Touch: Thermal Dissipation as a Sensing Modality
}

\author{
Hilary Emenike*, Farooq Dar*, Mohan Liyanage*, Rajesh Sharma*, Agustin Zuniga ${ }^{\dagger}$, \\ Mohammad A. Hoque ${ }^{\dagger}$, Marko Radeta ${ }^{\ddagger}$, , Petteri Nurmi ${ }^{\dagger}$ and Huber Flores* \\ *Institute of Computer Science, University of Tartu, Tartu, Estonia \\ ${ }^{\dagger}$ Department of Computer Science, University of Helsinki, Helsinki, Finland \\ $\ddagger$ Wave Lab, ITI/LARSyS, University of Madeira, Funchal, Portugal

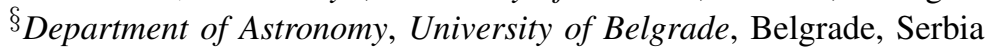 \\ firstname.lastname@ \{ut.ee, helsinki.fi, iti.larsys.pt $\}$
}

\begin{abstract}
We contribute MIDAS as a novel sensing solution for characterizing everyday objects using thermal dissipation. MIDAS takes advantage of the fact that anytime a person touches an object, it results in heat transfer. By capturing and modeling the dissipation of the transferred heat, e.g., through the decrease in the captured thermal radiation, MIDAS can characterize the object and determine its material. We validate MIDAS through extensive empirical benchmarks and demonstrate that MIDAS offers an innovative sensing modality that can recognize a wide range of materials - with up to $83 \%$ accuracy - and generalize to variations in the people interacting with objects.

Index Terms - thermal imaging, mobile computing, pervasive computing, IoT, material sensing
\end{abstract}

(C) 2021 IEEE. Personal use of this material is permitted. Permission from IEEE must be obtained for all other uses, in any current or future media, including reprinting/republishing this material for advertising or promotional purposes, creating new collective works, for resale or redistribution to servers or lists, or reuse of any copyrighted component of this work in other works.

\section{INTRODUCTION}

Every day humans touch numerous objects, ranging from their personal possessions to home appliances, food, clothing, and many other objects [1]. Capturing information about the objects people interact with has the potential to offer rich insights into human behaviour [2], ranging from simple everyday activity monitoring to more complex applications, such as dietary monitoring [3] or detection of household practices [4]. Unfortunately, capturing such information is fraught with difficulty as current solutions suffer from some significant limitations. Specifically, contact-based approaches, such as RFID, either require instrumenting all objects or keeping the sensing device in close contact with the object for a sufficiently long period [5], [6]. Non-contact based solutions, such as image-based object recognition, in turn, have limited discriminatory power and are vulnerable to the environment where they are operated [7]. For example, imagebased recognition is vulnerable to changes in illumination, camera angle, and picture resolution [8].

In this paper, we develop MIDAS as a novel sensing solution for characterizing everyday objects using the thermal dissipation resulting from human touch. MIDAS exploits the fact that anytime a person touches an object, it results in heat transfer. Over time, the transferred heat dissipates from the object as the object attempts to reach thermal equilibrium with the surrounding environment. The speed of this dissipation depends on the material characteristics of the object. MIDAS captures the heat transfer and the ensuing dissipation, and uses these to model and characterize the materials of the objects that the user interacts with. MIDAS captures the changes in heat using a commercial-off-the-shelf thermal camera, which helps us to overcome the key limitations of previous techniques. Specifically, thermal cameras can operate without requiring contact and being robust to illumination conditions and the overall capture context. Thermal imaging is also increasingly feasible thanks to affordable off-the-shelf thermal cameras.

We validate MIDAS through rigorous experiments that consider 14 different everyday objects that cover the most common materials used in manufactured products. As part of the experiments, we also demonstrate that MIDAS generalizes to human temperature variations by considering the robustness of thermal dissipation characteristics of objects with 18 different individuals. Our results indicate that human-emitted radiation can be used to characterize different materials and that this characterization is robust against variations in individuals and the way they interact with objects. MIDAS can determine the correct material with up to $83 \%$ accuracy, a $16 \%$ improvement on a computer vision baseline that uses deep learning. MIDAS offers a novel sensing modality that is highly useful for characterizing everyday objects, enabling a broad range of innovative applications.

\section{Feasibility Assessment}

We first conduct two preliminary studies to demonstrate that heat transfer from humans can be used to characterize different materials and household objects. We capture thermal radiation using an off-the-shelf smartphone (CAT S60) and validate the measurements using a thermometer scanner that serves as a reference instrument. In the following, all statistically significant differences were separately validated using measurements from the reference device. 


\section{A. Testbed}

We capture thermal footprints using two devices: a handheld thermal imaging scanner (FLIR TG267) and a Caterpillar smartphone (CAT S60) with an integrated FLIR thermal imaging. In all experiments, we place the smartphone on a tripod at a distance of $30 \mathrm{~cm}$ to $35 \mathrm{~cm}$ from the object. We performed manual calibration on the camera after it had attained thermal equilibrium with the environment - room temperature of $22{ }^{\circ} \mathrm{C}$ to $23.5^{\circ} \mathrm{C}$. The video was recorded with the CAT S60, while we took thermal reference photos with the TG267 scanner. The room's ambient temperature was measured using a Netatmo weather station ${ }^{1}$. Dissipation times were estimated automatically from the thermal video and validated by comparing against a ground truth obtained from a manual inspection of the video with a stopwatch.

\section{B. Plastic thermal footprint dissipation}

Experimental Design: We first measure the dissipation time of a thermal footprint in different plastic materials and correlate the captured footprint with the emissivity coefficient of the material. In this experiment, we focus solely on plastics to ensure the emissivity of the materials is known. In subsequent sections, we demonstrate that our solution generalizes to other materials. We consider the most common plastics that can be found in everyday objects: LDPE (Low Density Polyethylene), HDPE (High Density Polyethylene), PP (Polypropylene), PS (Polystyrene), and PVC (Polyvinyl chloride). The material of an object is specified by its Resin Identification Code (RIC), and all materials have well known emissivity coefficients $(\epsilon=0.90-0.97)$. The tested plastic samples have identical shape and size and have been produced by an identical manufacturing process $^{2}$. This ensures the samples' differences result from inherent material properties and are not an artifact of any external differences (e.g., shape or stiffness). In the experiments, we first place the plastic sample inside a fridge with a constant temperature of $5{ }^{\circ} \mathrm{C}$, to obtain a baseline temperature for comparison. To measure different temperatures, we use a constant heat source (lamp bulb of $60 \mathrm{~W}$ ) to heat the plastic samples. The lamp is placed at a fixed distance of $10 \mathrm{~cm}$ from the samples to avoid burn damage while ensuring they are exposed to sufficient amounts of thermal radiation. We consider different heating periods (1,2, 3 and 4 minutes) to correspond to differing initial temperatures and measure the dissipation of the thermal footprint. During the experiments, ambient temperature oscillated from $22^{\circ} \mathrm{C}$ to $24^{\circ} \mathrm{C}$.

Results: The results in Figure 2a show that the thermal footprint dissipation varies across the materials. The (Spearman) correlation between dissipation time and emissivity coefficient of the materials was found statistically significant $(\rho=0.66$, $\mathrm{p}<.05$ ), indicating that the dissipation characteristics indeed provide information about the material of the object.

\footnotetext{
${ }^{1}$ https://www.netatmo.com

${ }^{2}$ https://www.materialsampleshop.com/products/plastics-sample-set
}

\section{Other thermal footprint dissipations}

Testbed: We next demonstrate that the findings of the previous section generalize to other objects and materials by measuring the dissipation time of a thermal footprint on different household objects. We consider common household objects, shown in Figure 1, and include rubber, plastics, glass, ceramic, and metal. The considered objects include: a beer can (A), ceramic cup (B), takeaway box (C), plastic bottle (D), glass bottle (E), coffee cup (F), plastic cup (G), cigarette butt (H), glass jar (I), milk pack $(\mathrm{J})$, aluminum aerosol can $(\mathrm{K})$, rubber glove (L), steel spoon (M) and a face mask (N). Similar to our previous experiment, we analyze the thermal dissipation time after the objects are held for 1,2,3, and $4 \mathrm{~min}$. The average body temperature of the human subject holding the object ranged from $35^{\circ} \mathrm{C}$ to $36^{\circ} \mathrm{C}$, and the ambient temperature was from $22^{\circ} \mathrm{C}$ to $24^{\circ} \mathrm{C}$.

Results: The results in Figure 2 are in line with the results for the plastic objects and show that the dissipation times differ across the objects and materials. Friedman test using object materials as experimental condition showed the differences of the materials to be statistically significant $\left(\chi^{2}(2)=48.83\right.$, p $<.05, W=0.93)$, demonstrating that thermal radiation can characterize different object materials.

\section{MIDAS PIPELINE}

The results of the previous section demonstrated that dissipation of thermal footprints provides information that can be used to characterize different objects and identify their materials. We next briefly describe the sensing pipeline used by MIDAS, our sensing solution for characterizing everyday objects. MIDAS takes a sequence of thermal images taken from the object's surface as input and returns an estimate of the most likely material of that object.

Preprocessing: Commercial off-the-shelf thermal cameras are commonly based on uncooled FLIR (forward looking infrared) technology, and they suffer from inaccuracies resulting from the heating of the camera [9]. Other factors to influence measurement quality include misalignment between thermal and RGB pictures, internal recalibration of the camera, and low resolution. To mitigate these effects, we preprocess the thermal camera data by examining the background of consecutive images and removing images with significant dissimilarities. We also apply denoising on the images and normalize the thermal values to a consistent scale (between 0 and 255), which allows us to manipulate the images in gray scale.

Dissipation rate: We estimate the dissipation rate of the thermal footprint from the normalized sequence of pictures as the function of area reduction of the thermal footprint given by the equation: $R A=\left(A_{i}-A_{t}\right) / A_{i}$, where $R A$ is the reduction area percentage, $A_{i}$ is the initial area, and $A_{t}$ is the reduced target area [10] (see Figure 3). The reduction area between consecutive images is used to create vectors that model the dissipation time of thermal footprints for each object.

Implementation: Vectors (same length) with dissipation time are used as feature vectors and the type of object as label class. 


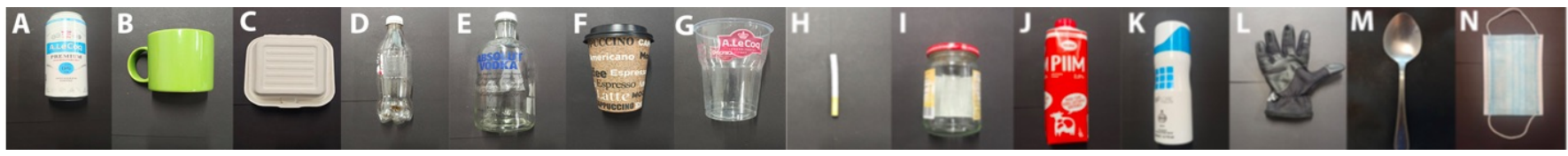

Fig. 1: Selected waste materials for preliminary experiments. A (Beer Can), B (Ceramic Cup), C (Takeaway Box), D (Plastic bottle), E (Glass Bottle), F (Coffee Cup), G (Plastic Cup), H(Cigarette Butt), I (Glass Jar), J (Milk pack), K (Aerosol Can), L (Rubber glove), M (Metal spoon), N (Face mask).

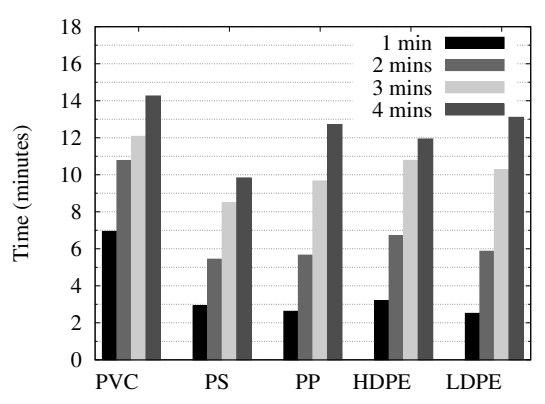

(a)

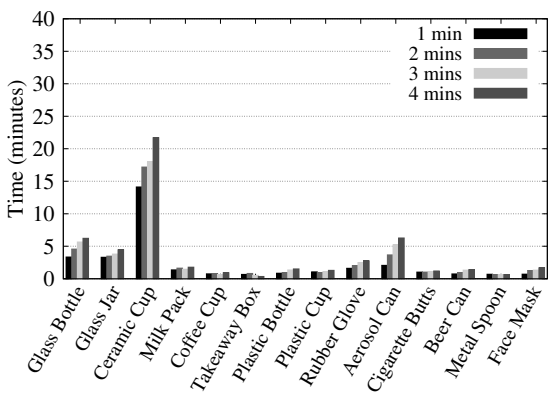

(b)



(c)

Fig. 2: Dissipation time of thermal footprints in different plastic materials and waste material using two different devices: (a) Grouped by RIC code, (b) Thermometer scanner FLIR TG267 (baseline), and (c) Smartphone CAT s60.

We then construct classification models using common ML techniques: Random Forests (RF), Support Vector Machines (SVM), and Multi-Layer Perceptron Classifier (MLPC); see Section V-C for comparison of these techniques.

\section{Robustness of Thermal DisSIPATION FOOTPRINTS}

The experiments shown in Sec. II demonstrated that the dissipation characteristics of thermal footprints vary across different materials. Human body temperature varies across individuals and even within the same individual at different times of day [11], which results in variations in the initial thermal footprints. Ensuring MIDAS can operate robustly against these variations is essential for ensuring the usefulness of MIDAS in practical use. In this section, we describe experiments where 18 different individuals touch everyday objects, and we use the resulting thermal footprints and their dissipation to characterize the materials of the objects. The measurement setup is described in Section II-A.

\section{A. Experimental Setup}

Experiment Design: We conduct a $3 \times 3$ within-subject design with holding pattern type and object type as independent variables. Both variables have three levels: Fixed-hold $(\mathrm{FH})$, Natural-hold $(\mathrm{NH})$ and Quick-hold $(\mathrm{QH})$ for the former and Plastic bottle (BOTTLE), Cardboard cup (CUP) and Cigarette butt (CIGAR) for the latter. To eliminate order effects, whilst keeping the number of combinations manageable, holding pattern type was fully counterbalanced, whereas object type was counterbalanced following a Latin Square design, resulting in nine experimental conditions: (1) BOTTLE-FH, (2) CUP-FH, (3) CIGAR-FH; (4) BOTTLE-NH, (5) CUP-NH, (6) CIGARNH; (7) BOTTLE-QH, (8) CUP-QH, and (9) CIGAR-QH.

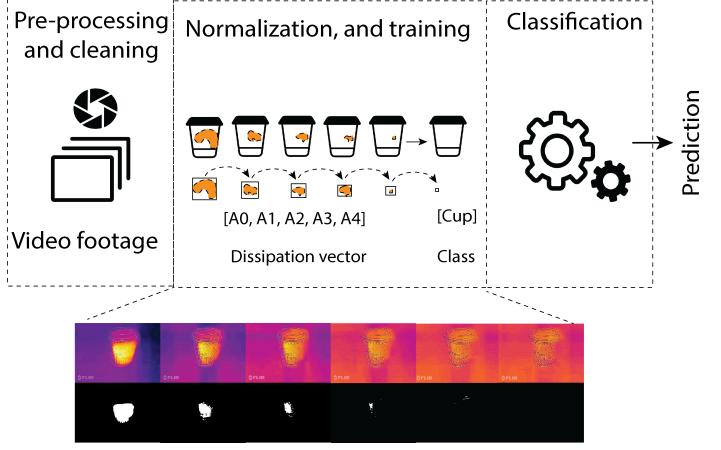

Fig. 3: Processing pipeline of material classification based on dissipation time of thermal footprints.

In the Fixed-hold condition, objects were grabbed and held from a specific static position for one minute. In the Naturalhold condition, objects were held freely for one minute, simulating usual everyday interactions with the object. For instance, a participant holds the empty bottle for one minute while looking for a trash bin. In the Quick-hold condition, objects were held by participants freely for a $10 \mathrm{~s}$ span.

Participants: We recruited a total of $\mathrm{N}=18$ participants (Males=9, Females=9) for the user study. Participants were students, admin staff and professionals from different fields, and nationalities, with little or no knowledge about thermal imaging. Their average age was $28 \pm 7.8$ years.

Task: Participants were asked to hold objects and to simulate normal interactions with them. To produce data for natural interaction, we also asked the participants to contextualize a normal interaction context. When interacting with the BOTTLE, participants were asked to simulate drinking from the bottle and then looking for a trash bin to dispose an empty 
bottle. Similarly, participants were asked to stand while engaging in a short conversation with an acquaintance/friend for the CUP. Finally, for the CIGAR condition, participants were asked to simulate taking a cigarette from a cigarette box and then holding the cigarette from the filter while asking for a light. The cigarette was not lighted during the experiment.

Procedure: Before starting the experiment, each participant was invited to relax on a comfortable chair for 10 minutes to enable the body temperature to acclimatize to the room's ambient temperature, which oscillated around $22{ }^{\circ} \mathrm{C}$ to $23.5^{\circ} \mathrm{C}$ throughout the experiments. During this period, participants received a brief explanation of the study and signed an informed consent form, following local IRB regulations. Once the participant was ready to start the experiment, his/her body temperature was measured from the forehead using a clinically certified contactless optical thermometer (DR CHECK FC500). The nine experimental conditions were presented to participants. In each condition, the object was first placed inside an empty fridge with a temperature of $5{ }^{\circ} \mathrm{C}$ for one minute. This procedure rules out residual thermal radiation in the material between experiments and provides the material with a baseline temperature to make our results comparable across the participants.

Kitchen tongs were used to take the object from the fridge to avoid heat transfer from humans to objects. Next, the object was placed on a table for one minute to adapt it to the ambient temperature. After that, participants carried out the corresponding experimental condition. Once finished, participants placed the object on a fixed marker drawn on a table with a black background and surface. The researcher conducting the study then used the CAT S60 to record video footage of the dissipation of the object's thermal footprint. In parallel, a thermometer scanner took thermal photos to serve as a reference baseline. A black background helps to obtain clean video footage of thermal footprint without any thermal influence from objects in the surrounding environment. At the end of the experiment, we measured the participant's temperature from palm and finger to the objects using the thermal imaging scanner. The evaluation took place in one university room across two weeks in time slots between 11:00 and to 07:00 pm. Since human temperature varies during the day [11], we considered only those times as they coincide with the working hours of the participants. For each participant, the overall experiment lasted $40 \mathrm{~min}$ to $45 \mathrm{~min}$.

\section{B. Baselines}

As part of the experiments, we compare the recognition performance of MIDAS to two state-of-the-art techniques: deep learning based automated computer vision [12], [13] and optical sensing [14].

Computer vision: We train a state-of-the-art Convolutional Neural Network (CNN) model using the publicly available TrashNet dataset [15]. We focus exclusively on the plastic materials category, which contains 626 images of plastic objects for training the deep learning model. Plastics are malleable, so their accurate recognition is very sensitive to changes. The dataset has images where the individual pieces are shown against a white background. As such images do not match realistic recognition settings, we supplement the dataset with an additional 767 images from the Japan Agency for Marine Earth Science and Technology (JAMSTEC) Deep-sea Debris Database dataset. We annotated the collected images manually by drawing a rectangle box around the object material in images. We labeled the TrashNet plastic items as "trash" and the JAMSTEC plastic items as "plastic". Both datasets were augmented by adding noise, hue, blue, horizontal flip, and vertical flip modifications to each original image, resulting in a total of 6985 images for model training input. We created and trained the PlasticNet model using Google Collab server GPU, with $100 \mathrm{k}$ iterations and a batch size of 12, running TensorFlow Lite 1.15. We used ssd_mobilenetv2_oidv4 for the base training model.

Light reflectivity: As our second baseline, we consider reflectivity analysis of materials [14] using a photoresistor connected to the analog input pin of an Arduino MEGA ADK. The photoresistor captures light changes based on its resistance exposure to the light intensity of the reflected material. As a light source, we rely on a red laser diode (wavelength $650 \mathrm{~nm}$ ). The object was located $2 \mathrm{~cm}$ away from the light source, depicting a practical usage of the sensor in transport belts and smart bins [12]. We took measurements with sensor for different materials (selection is described in Section II), for one minute from two different random places in the object.

\section{Results}

We next demonstrate that MIDAS can characterize different object materials accurately using the measurements from the controlled user evaluation described in the previous section. We consider robustness against variations in the way humans interact with objects and variations in individuals, and the overall classification performance for different materials.

\section{A. Differences in Thermal Footprints}

We first examine differences in thermal transfer from humans and the ensuing dissipation in the different objects. We separately consider the impact of the object and the hold type on thermal transfer.

The dissipation times of the three objects under the different hold type conditions are shown in Figure 4. From the figure, we can observe the differences in objects to be consistently different regardless of the hold type. Friedman Test using dissipation time and objects as experimental conditions shows the differences in objects to be significant for all of the three hold-type conditions: Fixed-hold $\left(\chi^{2}(2)=20.33, \mathrm{p}<.05\right.$, $W=0.56)$, Natural-hold $\left(\chi^{2}(2)=30.33, \mathrm{p}<.05, W=0.84\right)$ and Quick-hold $\left(\chi^{2}(2)=25.04, \mathrm{p}<.05, W=0.64\right)$. Pairwise post hoc comparisons using Wilcoxon test (with Bonferroni correction for multiple comparisons) confirmed that the differences in dissipation times are statistically significant for all object pairs across the three hold types.

We next assess how the hold type affects the thermal footprints. Friedman test using dissipation time of each object 
and the three experimental conditions shows hold type to result in significant differences for the plastic bottle $\left(\chi^{2}(2)=12.44\right.$, $\mathrm{p}<.05, \mathrm{~W}=0.34)$ and the cardboard cup $\left(\chi^{2}(2)=16.48\right.$, $\mathrm{p}<.05, \mathrm{~W}=0.45)$, but not for the cigarette butt. Post hoc comparisons indicate the thermal footprints for the Quickhold pattern to significantly differ from those in the Fixedand Natural- hold conditions. Results imply that differences in thermal footprints contain significant variation across objects regardless of how users touch or interact with them. Still, the dissipation times are impacted by the time the user holds the item. This is expected, as the time the user touches the object affects the extent of heat that can transfer and thus controls dissipation speed.

We also separately analyzed whether differences in body temperature across different body parts can affect the thermal footprint by comparing the thermometer results across the three measurement locations (forehead, hand palm, finger tips). Friedman test using part of body as experimental conditions showed significant differences for temperature $\left(\chi^{2}(2)=\right.$ 29.66, $p<.05, W=0.82$ ). Posthoc comparisons (DunnBonferroni) verified that the differences were statistically significant $(p<.01)$ between forehead and hand-palm and between forehead and finger-tips. The average temperature for the different parts of body were: forehead $36.33^{\circ} \mathrm{C}$, hand-palm $30.16^{\circ} \mathrm{C}$ and finger-tips $30.87^{\circ} \mathrm{C}$. These results show that fingers and hand palm generally induce similar heat transfer, further validating the robustness of the thermal footprints against the way people interact with the objects. The difference to forehead temperature, in turn, suggests that the hand palm and fingertips react to ambient temperature instead of correlating with the internal body temperature - as is the case for the forehead measurements. We note that the higher sensitivity of finger tips and hand palm also means that it can be potentially used to extract insights about human activity. For example, interactions with a smartphone can result in heat caused by battery temperature to transfer into the hand, and the contents of a hot drink can similarly affect the temperature.

Overall, the results indicate that human touch transfers a sufficient amount of heat, making it possible to characterize equipment based on touch without resorting to specialized technology. However, the results show that the dissipation times are affected by the time the user interacts with the objects - and other factors as will be shown in the next subsection - implying that relative differences in dissipation characteristics should be used instead of the exact dissipation times for characterizing materials.

\section{B. Other factors that influence thermal dissipation}

We thus far showed that the characterization of object materials with thermal radiation depends on exposure time, but not on the location where the object is being touched at. In this section, we investigate other factors that influence the transfer of thermal radiation from the human body to objects.

Gender and temperature: Body temperature generally influences thermal radiation transferred to objects. We next analyze whether the gender of the participants affects thermal transfer, i.e., whether there are significant differences in thermal transfer between female and male participants. We separately assessed effects for the hand palm and finger tips. KruskalWallis tests, using gender and part of the body as experimental conditions, show that there are significant differences in thermal transfer only for finger tips $\left(\chi^{2}(2)=5.08, \mathrm{p}<.05\right)$. This is most likely the result of differences in the size of the contact area with males typically having larger fingertip size [16].

When using gender and objects as experimental conditions, Kruskal-Wallis tests show there to be significant differences in the dissipation time for all three objects: cigarette butt $\left(\chi^{2}(2)=3.94, \mathrm{p}<.05\right)$, plastic bottle $\left(\chi^{2}(2)=12.17, \mathrm{p}\right.$ $<.05)$ and cardboard cup $\left(\chi^{2}(2)=7.75, \mathrm{p}<.05\right)$. These results indicate that the thermal footprint's dissipation time depends on temperature and that it is possible to identify whether a female or male individual has touched the object. While this result does not change the fact that objects can be characterized with thermal radiation, it is essential to highlight that thermal radiation can disclose additional information about the humans interacting with the objects. For example, it could be possible to use thermal footprints to compare household waste sorting practices between genders.

External temperature of ambient environment: Surrounding temperature of the object directly influences the dissipation time of the thermal footprint. We quantity the influence of this factor through additional small scale experiments. First, the BOTTLE was held by a human hand for different periods at an ambient temperature of $22{ }^{\circ} \mathrm{C}$ to $23.5^{\circ} \mathrm{C}$. Next, the BOTTLE was placed inside a colder environment (fridge with a temperature of $5{ }^{\circ} \mathrm{C}$ ). We then measured the dissipation time of the thermal footprint when changing from ambient to colder environment using both the CAT S60 and the thermometer scanner. Figure 5 shows the results. We also include the thermal footprint's dissipation time in the ambient environment for comparison purposes (baseline). We notice the total dissipation time of the thermal footprint is halved when changing to a colder environment. Still, overall the differences in change patterns remain consistent for the different objects. This suggests that the environment affects the fingerprints. Note that the magnitude of this change is proportional to heat difference and impacts all objects equally. Hence, incorporating the ambient temperature in the thermal dissipation footprints is sufficient to overcome potential issues from differing temperatures.

Internal temperature absorbed from contents: Besides the ambient temperature of the surrounding environment, objects can also be influenced by the thermal radiation resulting from the contents of the object. For example, a cardboard cup can be filled with a hot or a cold drink. To investigate this further, we fill the BOTTLE with water with a temperature of $21.2^{\circ} \mathrm{C}$ to $21.5^{\circ} \mathrm{C}$. Before the experiment, we place the BOTTLE inside a fridge $\left(5^{\circ} \mathrm{C}\right)$ to eliminate thermal radiation carryover effects between experiments. We then compare the dissipation times of an empty and filled bottle in ambient temperature 


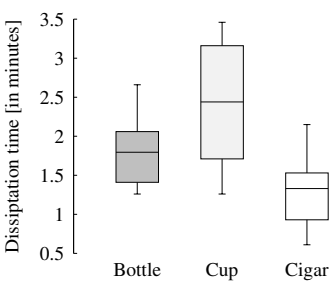

(a) Fixed-hold



(b) Natural-hold



(c) Quick-hold

Fig. 4: Thermal transferred conditions applied over three different objects (plastic bottle, cardboard cup, cigarette but)



(a) CAT s60

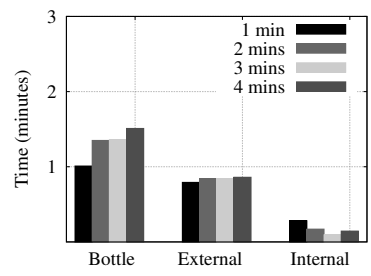

(b) Thermometer Scanner
Fig. 5: Influence of internal and external temperatures.

$\left(22^{\circ} \mathrm{C}\right.$ to $\left.23.5^{\circ} \mathrm{C}\right)$. Figure 5 shows the results. We observe that the internal radiation impacts the thermal footprint of the BOTTLE. This is relevant to identify end-products that have not been fully consumed. In practice, such cases should be modeled as a separate (mixed) object to ensure the model can distinguish the pure material from those cases where there are no contents inside the object. Compared with the thermal footprint of the empty BOTTLE, we can observe that the thermal footprint of the filled water BOTTLE dissipates faster as a result of the larger difference with the environment.

Distance between object and thermal camera: In the experiments thus far, the distance between the thermal camera and the objects has been fixed at $30 \mathrm{~cm}$ to $35 \mathrm{~cm}$ (baseline). We next analyze the effect of longer distances by considering three additional distances: $70 \mathrm{~cm}$ (distance-1), $105 \mathrm{~cm}$ (distance2) and $210 \mathrm{~cm}$ (distance-3). We focus exclusively on the BOTTLE with a Fixed-hold setup of one minute. At $70 \mathrm{~cm}$ distance, the dissipation time does not change significantly (average time $=1.13 \mathrm{~min}$ ) for the CAT S60. At longer distances, we observed higher variations in the dissipation time with the CAT S60. At $105 \mathrm{~cm}$ distance the average time was 0.76 minutes and at $210 \mathrm{~cm}$ the average time was 0.26 minutes. The resolution of the thermal camera in the CAT S60 is $80 \times 60$, which seems to be sufficient for up to a meter. The thermometer scanner has a higher resolution $160 \times 120$, but a slower frame rate $(6.67 \mathrm{~Hz}$ vs. $8 \mathrm{~Hz})$. For a $210 \mathrm{~cm}$ distance, the thermometer fails to observe a proper thermal footprint. This suggests that a higher resolution is not guaranteed to extend the operational range of MIDAS as also the frame rate needs to be considered.

Temperature sensitivity: We next analyze the sensitivity of the dissipation times to slight variations in temperature. To accomplish this, we further analyze the BOTTLE material in the fixed-hold condition, as above. We use a JANOEL18S incubator with adjustable temperature to achieve controlled changes in the temperature. We put the object inside the incubator at a constant temperature for $15 \mathrm{~min}$ to ensure that the entire object has exactly the same temperature. We expose the material to temperatures ranging from $36^{\circ} \mathrm{C}$ to $39^{\circ} \mathrm{C}$, corresponding to normal and elevated temperature levels in a human. After the object was heated up, it was transferred to the testbed that was used to record footage. We then proceed to measure the dissipation time of the thermal footprint. The room temperature that the object had to acclimatize ranges from $23^{\circ} \mathrm{C}$ to $23.5^{\circ} \mathrm{C}$. The dissipation times for the different temperatures were: $3.33 \mathrm{~min}$ for $36^{\circ} \mathrm{C}, 3.73 \mathrm{~min}$ for $37^{\circ} \mathrm{C}$, $4.23 \mathrm{~min}$ for $38^{\circ} \mathrm{C}$, and $4.34 \mathrm{~min}$ for $39^{\circ} \mathrm{C}$ respectively. The dissipation time is expected to be a function of the temperature difference between the object and the environment, and our results confirm that these subtle temperature differences can be captured robustly using a commercial-off-the-shelf thermal camera. While accurate human temperature is not possible to estimate, we envision that our approach can be used to detect abnormalities in human temperatures. For instance, instead of monitoring the face of people with thermal cameras at an airport, it could be possible to detect abnormal temperatures by monitoring the tangible objects that people touch while passing a security check.

\section{Dissipation time classification performance}

We next demonstrate that our approach can support the coarse-grained classification of object materials based on the dissipation time of thermal footprints - and other contextual factors. As described in Section III, we considered three classification techniques: Random Forest (RF), Support Vector Machine (SVM), and Multi-Layer Perceptron (MLP). The results of the classification experiments are shown in Table I. When only the thermal footprint is available, the highest classification accuracy for material detection is approximately $83 \%$. Incorporating information about the hold pattern type does not improve the results, suggesting that the way people grasp the materials does not impact performance. In contrast, when having information about whether the person is male or female, we can observe that the accuracy to predict materials improves up to $86 \%$. Similarly, when attempting to predict whether the user is male/female, we can observe that dissipation time and material information provide a high accuracy estimation of around $78 \%$. 
TABLE I: Material classification accuracy (\%) in different experimental conditions. Model data $\rightarrow$ Predicted. Classification Method: Random Forest (RF), Support Vector Machine (SVM), Multi-layer Perceptron (MLPC).

\begin{tabular}{lcccc}
\hline Test & RF & SVM & MLPC & Average \\
\hline Predicting Material (M) & & & & \\
(Vector) $\rightarrow$ M & 90.9 & 77.3 & 81.8 & $\mathbf{8 3 . 3}$ \\
(Vector, Context) $\rightarrow$ M & 90.9 & 77.3 & 81.8 & $\mathbf{8 3 . 3}$ \\
(Vector, Gender) $\rightarrow$ M & 90.9 & 86.4 & 81.8 & $\mathbf{8 6 . 4}$ \\
(Vector, Context, Gender) $\rightarrow$ M & 86.4 & 81.8 & 81.8 & $\mathbf{8 3 . 3}$ \\
$\quad$ Average & 89.8 & 80.7 & 81.8 & $\mathbf{8 4 . 1}$ \\
Predicting Context, Gender & & & & \\
(Material, Vector) $\rightarrow$ Context & 77.3 & 81.8 & 72.7 & $\mathbf{7 7 . 3}$ \\
(Material, Vector) $\rightarrow$ Gender & 77.3 & 77.3 & 81.8 & $\mathbf{7 8 . 8}$ \\
$\quad$ Average & 77.3 & 79.6 & 77.3 & $\mathbf{7 8 . 1}$ \\
\hline
\end{tabular}



(a)

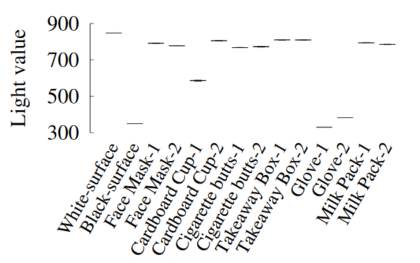

(b)
Fig. 6: Baselines, a) Trained model PlasticNet, discriminating plastics against other object materials, b) Light reflectivity values of different materials measured with a photo-resistor.

\section{Comparison with other approaches}

Finally, we compare MIDAS against the two baselines: computer vision and optical sensing. We test computer vision using 31 images depicting real tossed plastic objects [17]. In total, 33 separate plastic items were present in the 31 images. The deep learning model managed to identify 23 of the 33 items (69.7\% in accuracy). Figure 6 highlights the main limitation of the model, being unable to identify multiple objects simultaneously and objects whose shape changes drastically. As the vision-based approach does not extract any internal characteristics of the objects, the only way to use it for material recognition is to map specific item types to materials (e.g., drink bottles are typically made of PET). This approach works reasonably when the objects are sufficiently distinctive but fails in everyday use cases where the shape of the objects has changed, e.g., tossed objects often have lost their original shape, and other visual characteristics may similarly have undergone significant changes.

Figure $6 \mathrm{~b}$ shows the results for the second baseline, light reflectivity. The low variation in reflectivity values indicates that light can accurately characterize different materials. However, we also observe that different parts of the same material can be characterized very differently, e.g., Cardboard Cup-1 and Cardboard Cup-2. This is because objects comprise of different materials and colors, which can affect reflectance. Another limitation of this approach is the need for the sensors to contact the material to classify it accurately.

\section{DISCUSSION}

Human temperature: Human temperature changes in cycles, being at its highest during hours of activity (day) and lowest during sleep (night) [11]. We demonstrate that interactions with objects can be used to characterize materials. The best results are obtained when the body temperature is stable, but the relative differences in thermal footprints are consistent across variations in body temperature. Conversely, interactions with an object of known material and in a stable environment can be used to detect relative differences in body temperature.

Room for improvement: Adapting our approach to continuous monitoring requires accurate and noise-free thermal images, e.g., using calibration [9]. Not all materials can be characterized using our solution as thermal cameras have different emissivity ranges and some materials may reflect too much - or too little - thermal radiation. These materials are usually used to preserve user's privacy, e.g., ATM pin codes [18]. While outside the scope of our present work, there is also room for developing application areas, e.g., validating temperature differences with patients as part of clinical studies.

Other material properties: Dissipation time of thermal footprint gives insights about material types and correlates with emissivity. Thermal imaging could be used to potentially infer other material properties, such as thickness and elasticity. Potential use cases include detecting the pollutant type of marine plastics [19] and monitoring the decay in organic materials using differences in thermal dissipation characteristics.

Robots and autonomous devices: Thermal radiation analysis of objects touched by humans can be used to inform and train different robots and autonomous devices about the material properties of objects. New sensing and interaction modalities can also be envisioned as part of robotic systems, e.g., incorporating heat sensation to detect the material of an object and to enable autonomous devices to adjust their operations with objects in the surrounding environment [20].

Augmented reality systems: Augmented reality systems that mix the real and virtual worlds can benefit from thermal radiation monitoring. By piggybacking the human-touch on objects, it is possible to recognize the materials of objects further. These objects are mapped to the virtual world by considering their inherent natural material properties, such as wood, concrete, glass, and plastic.

\section{RELATED WORK}

Thermal imaging: The usage of thermal imaging has been studied in different domains and applications with examples ranging from monitoring the manufacturing process of smartphone hardware components [21] to medical analysis [22], [23]. Other examples include facial recognition for bio-metric authentication [24], cognitive analysis [25], gestures [26], [27], and energy modeling of IoT devices [28]. Our work extends thermal imaging to material classification.

Material sensing: Materials have different characteristics different properties that can be exploited to categorize them. The most common material sensing approach is to rely on different 
parts of the light spectrum and measure either reflection or absorption at different frequencies. Examples range from the use of green light sensing to detect plastic waste [29] to the use of near-infrared sensing to facilitate medicine adherence [30] and the use of hyperspectral imaging for estimating sugar content in drinks [31]. Also, deep learning approaches for detecting different material types from reflection patterns at different wavelengths have been proposed [32]. Our work extends these by using thermal radiation in the infrared spectrum to estimate internal characteristics of materials through heat dissipation.

Sensorless sensing: Wireless signals can also be used to identify properties in materials. Examples include the use of variations in WiFi signal propagation characteristics to identify liquids [33], and the use of surface tension to characterize liquids [34], [35]. These methods generally require either close contact with the material or a transmitter - receiver pair to be placed on opposite sides of the material. Our work offers a non-contact technique for material characterisation that piggybacks thermal radiation generated from humans.

\section{SUMMARY AND CONCLUSIONS}

We developed MIDAS as an innovative sensing solution for characterizing and recognizing everyday objects from the dissipation of residual thermal energy resulting from human touch. MIDAS uses thermal imaging to monitor thermal energy changes over time and models these changes to infer and characterize the material type of an object. Through extensive empirical benchmarks, we demonstrated that changes in thermal footprints are robust to variations in the way people interact with objects and the people themselves. MIDAS recognizes different material with up to $83 \%$ accuracy using only the dissipation of thermal footprints. Our solution offers an innovative sensing solution for classifying materials and taking advantage of human interactions with everyday objects.

\section{ACKNOWLEDGMENT}

This research is supported by the European Regional Funds through the IT Academy Programme. We would like to thank our shepherd Prof. Paul Lukowicz and the anonymous reviewers for feedback. 


\section{REFERENCES}

[1] P. Zuccotti, Every Thing We Touch: A 24-hour Inventory of Our Lives. Penguin UK, 2015.

[2] R. L. Klatzky, S. J. Lederman, and V. A. Metzger, "Identifying objects by touch: An "expert system"," Perception \& psychophysics, vol. 37, no. 4, pp. 299-302, 1985.

[3] S. Bi, T. Wang, N. Tobias, J. Nordrum, S. Wang, G. Halvorsen, S. Sen, R. Peterson, K. Odame, K. Caine et al., "Auracle: Detecting eating episodes with an ear-mounted sensor," Proceedings of the ACM on Interactive, Mobile, Wearable and Ubiquitous Technologies, vol. 2, no. 3, pp. 1-27, 2018.

[4] A. A. Babaei, N. Alavi, G. Goudarzi, P. Teymouri, K. Ahmadi, and M. Rafiee, "Household recycling knowledge, attitudes and practices towards solid waste management," Resources, Conservation and Recycling, vol. 102, pp. 94-100, 2015.

[5] U. Ha, Y. Ma, Z. Zhong, T.-M. Hsu, and F. Adib, "Learning food quality and safety from wireless stickers," in Proceedings of the 17th ACM Workshop on Hot Topics in Networks, 2018, pp. 106-112.

[6] J. Wang, J. Xiong, X. Chen, H. Jiang, R. K. Balan, and D. Fang, "Tagscan: Simultaneous target imaging and material identification with commodity rfid devices," in Proceedings of the 23rd Annual International Conference on Mobile Computing and Networking, 2017, pp. 288-300.

[7] H.-S. Yeo, J. Lee, A. Bianchi, D. Harris-Birtill, and A. Quigley, "Specam: Sensing surface color and material with the front-facing camera of a mobile device," in Proceedings of the 19th International Conference on Human-Computer Interaction with Mobile Devices and Services, 2017, pp. 1-9.

[8] G. Schwartz and K. Nishino, "Recognizing material properties from images," IEEE transactions on pattern analysis and machine intelligence, vol. 42, no. 8, pp. 1981-1995, 2019.

[9] T. Malmivirta, J. Hamberg, E. Lagerspetz, X. Li, E. Peltonen, H. Flores, and P. Nurmi, "Hot or not? robust and accurate continuous thermal imaging on flir cameras," in 2019 IEEE International Conference on Pervasive Computing and Communications (PerCom. IEEE, 2019, pp. $1-9$.

[10] N. C. of Examiners for Engineering, Fundamentals of Engineering: Supplied-reference Handbook. Kaplan AEC Engineering, 2003.

[11] Z. Obermeyer, J. K. Samra, and S. Mullainathan, "Individual differences in normal body temperature: longitudinal big data analysis of patient records," Bmj, vol. 359, 2017.

[12] G. White, C. Cabrera, A. Palade, F. Li, and S. Clarke, "Wastenet: Waste classification at the edge for smart bins," arXiv preprint arXiv:2006.05873, 2020.

[13] V. Ruiz, Á. Sánchez, J. F. Vélez, and B. Raducanu, "Automatic imagebased waste classification," in International Work-Conference on the Interplay Between Natural and Artificial Computation. Springer, 2019, pp. 422-431.

[14] A. Singh, P. Aggarwal, and R. Arora, "Iot based waste collection system using infrared sensors," in 2016 5th International Conference on Reliability, Infocom Technologies and Optimization (Trends and Future Directions)(ICRITO). IEEE, 2016, pp. 505-509.

[15] R. A. Aral, Ş. R. Keskin, M. Kaya, and M. Hacıömeroğlu, "Classification of trashnet dataset based on deep learning models," in 2018 IEEE International Conference on Big Data (Big Data). IEEE, 2018, pp. 2058-2062.

[16] M. A. Acree, "Is there a gender difference in fingerprint ridge density?" Forensic science international, vol. 102, no. 1, pp. 35-44, 1999.

[17] J. C. Prata, A. L. Silva, T. R. Walker, A. C. Duarte, and T. RochaSantos, "Covid-19 pandemic repercussions on the use and management of plastics," Environmental Science \& Technology, vol. 54, no. 13, pp. 7760-7765, 2020.

[18] Y. Abdelrahman, M. Khamis, S. Schneegass, and F. Alt, "Stay cool! understanding thermal attacks on mobile-based user authentication," in Proceedings of the 2017 CHI Conference on Human Factors in Computing Systems, 2017, pp. 3751-3763.

[19] H. Flores, N. H. Motlagh, A. Zuniga, M. Liyanage, M. Passananti, S. Tarkoma, M. Youssef, and P. Nurmi, "Toward large-scale autonomous marine pollution monitoring," IEEE Internet of Things Magazine, 2021.

[20] G. Li, S. Liu, L. Wang, and R. Zhu, "Skin-inspired quadruple tactile sensors integrated on a robot hand enable object recognition," Science Robotics, vol. 5, no. 49, 2020.
[21] Q. Xie, M. J. Dousti, and M. Pedram, "Therminator: A thermal simulator for smartphones producing accurate chip and skin temperature maps," in IEEE/ACM International Symposium on Low Power Electronics and Design (ISLPED), Aug 2014, pp. 117-122.

[22] G. Sun, S. Abe, O. Takei, and T. Matsui, "A portable screening system for onboard entry screening at international airports using a microwave radar, reflective photo sensor and thermography," in 2011 2nd International Conference on Instrumentation, Communications, Information Technology, and Biomedical Engineering. IEEE, 2011, pp. 107-110.

[23] B. Harangi, T. Csordás, and A. Hajdu, "Detecting the excessive activation of the ciliaris muscle on thermal images," in 2011 IEEE 9th International Symposium on Applied Machine Intelligence and Informatics (SAMI). IEEE, 2011, pp. 329-331.

[24] M. K. Bhowmik, D. Bhattacharjee, M. Nasipuri, D. K. Basu, and M. Kundu, "Classification of polar-thermal eigenfaces using multilayer perceptron for human face recognition," in 2008 IEEE Region 10 and the Third international Conference on Industrial and Information Systems. IEEE, 2008, pp. 1-6.

[25] Y. Abdelrahman et al., "Cognitive heat: Exploring the usage of thermal imaging to unobtrusively estimate cognitive load," Proc. ACM Interact. Mob. Wearable Ubiquitous Technol., vol. 1, no. 3, pp. 33:1-33:20, 2017.

[26] E. Larson, G. Cohn, S. Gupta, X. Ren, B. Harrison, D. Fox, and S. Patel, "Heatwave: thermal imaging for surface user interaction," in Proceedings of the SIGCHI Conference on Human Factors in Computing Systems, 2011, pp. 2565-2574.

[27] Y. Abdelrahman, A. Sahami Shirazi, N. Henze, and A. Schmidt, "Investigation of material properties for thermal imaging-based interaction," in Proceedings of the 33rd Annual ACM Conference on Human Factors in Computing Systems, 2015, pp. 15-18.

[28] H. Flores, J. Hamberg, X. Li, T. Malmivirta, A. Zuniga, E. Lagerspetz, and P. Nurmi, "Evaluating energy-efficiency using thermal imaging," in Proceedings of the 20th International Workshop on Mobile Computing Systems and Applications, 2019, pp. 147-152.

[29] H. Flores, A. Zuniga, N. H. Motlagh, M. Liyanage, M. Passananti, S. Tarkoma, M. Youssef, and P. Nurmi, "Penguin: aquatic plastic pollution sensing using auvs," in Proceedings of the 6th ACM Workshop on Micro Aerial Vehicle Networks, Systems, and Applications, 2020, pp. $1-6$.

[30] S. Klakegg, J. Goncalves, C. Luo, A. Visuri, A. Popov, N. van Berkel, Z. Sarsenbayeva, V. Kostakos, S. Hosio, S. Savage et al., "Assisted medication management in elderly care using miniaturised near-infrared spectroscopy," Proceedings of the ACM on Interactive, Mobile, Wearable and Ubiquitous Technologies, vol. 2, no. 2, pp. 1-24, 2018.

[31] W. Jiang, G. Marini, N. van Berkel, Z. Sarsenbayeva, Z. Tan, C. Luo, X. He, T. Dingler, J. Goncalves, Y. Kawahara et al., "Probing sucrose contents in everyday drinks using miniaturized near-infrared spectroscopy scanners," Proceedings of the ACM on Interactive, Mobile, Wearable and Ubiquitous Technologies, vol. 3, no. 4, pp. 1-25, 2019.

[32] Y. Cho, N. Bianchi-Berthouze, N. Marquardt, and S. J. Julier, "Deep thermal imaging: Proximate material type recognition in the wild through deep learning of spatial surface temperature patterns," in Proceedings of the 2018 CHI Conference on Human Factors in Computing Systems, 2018, pp. 1-13.

[33] A. Dhekne, M. Gowda, Y. Zhao, H. Hassanieh, and R. R. Choudhury, "Liquid: A wireless liquid identifier," in Proceedings of the 16th Annual International Conference on Mobile Systems, Applications, and Services, 2018, pp. 442-454.

[34] S. Yue and D. Katabi, "Liquid testing with your smartphone," in Proceedings of ACM MobiSys 2019. ACM, 2019, pp. 275-286.

[35] M. Wei, S. Huang, J. Wang, H. Li, H. Yang, and S. Wang, "The study of liquid surface waves with a smartphone camera and an image recognition algorithm," European Journal of Physics, vol. 36, no. 6, p. 065026, 2015. 